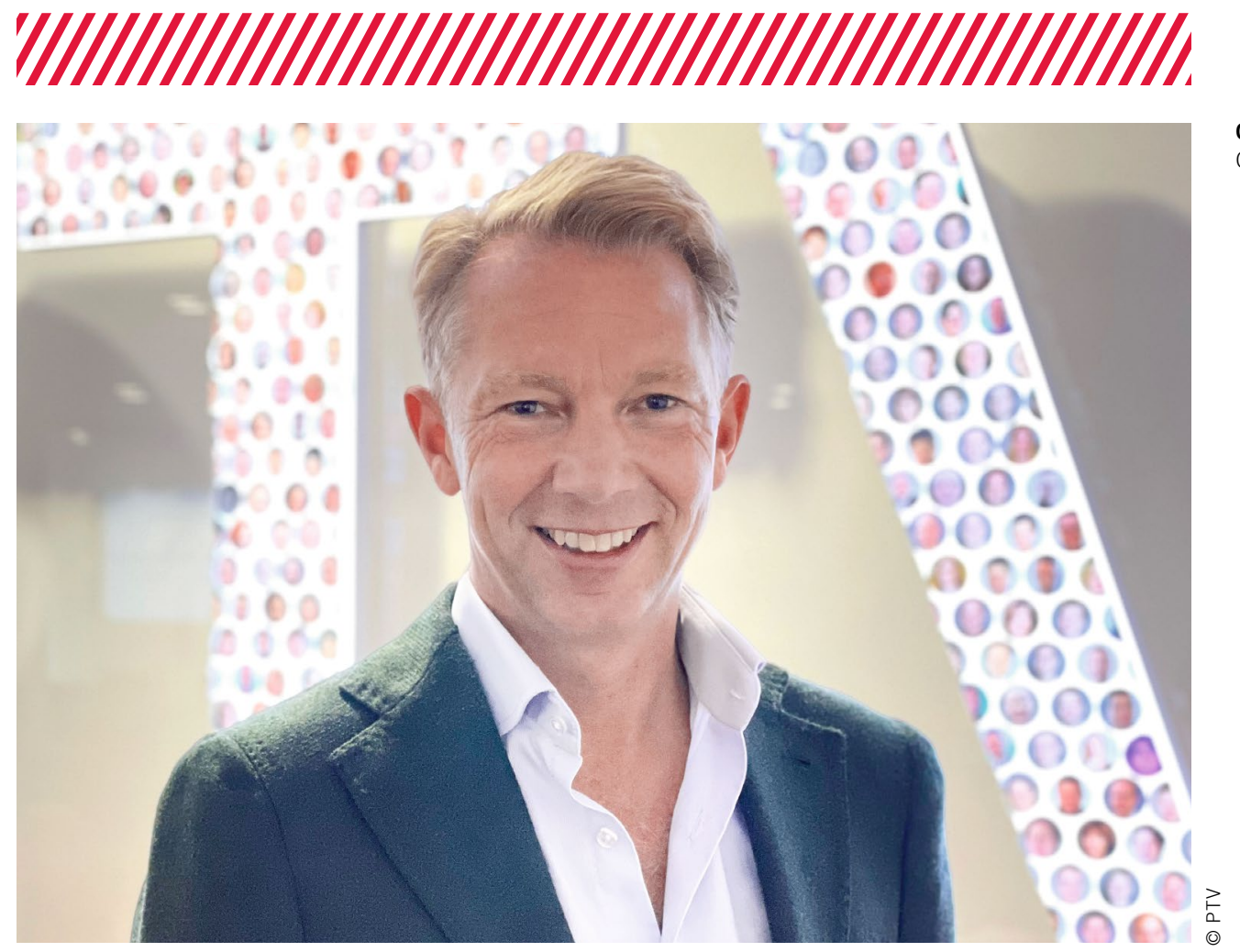

Christian U. Haas

CEO of the PTV Group

\title{
Autonomous Vehicles - Stress Test for the Urban Traffic Infrastructure?
}

Many players such as the US tech giants Waymo, Cruise and Tesla are working on turning the dream of autonomous driving into a reality. But Autonomous Vehicles (AVs) are only likely to catch on slowly and mainly in urban areas. Researchers expect that there will be a mix of conventional and autonomous cars on the roads until late in the $21^{\text {st }}$ century.

Analyses and research projects such as the EU-funded Coexist project are already providing information about the way in which AVs will operate. The assumption is that robotic cars will drive particularly cautiously in the early days. In order to guarantee the highest possible level of safety, they will leave longer distances between other vehicles, for example, and follow the speed limits more closely than human drivers. If the volume of traffic on the roads remains the same, this could result in the existing urban traffic infrastructure being pushed to its limits. Using a model of the university district of Cologne, we were able to demonstrate that in a scenario where cautious autonomous vehicles make up half of the traffic, the number of stops by all vehicles in urban traffic will increase by up to $80 \%$. The time wasted in traffic jams will rise by $29 \%$.

As AVs become more widespread, they are likely to band together in groups, known as platoons, in urban areas and this will allow them to travel more efficiently and to take up less space.

In order to counteract the increasing impact on the infrastructure and congestion in the transition period, cities will have to develop new solutions quickly. These will mainly relate to connectivity and will include, for example intersections and traffic lights that can communicate with AVs and enable them to adapt their behavior. It would also be possible to keep autonomous and manual vehicles separate, for example by allowing AVs to use bus lanes.

To ensure that AVs do not lead to an increased use of space and more traffic, they must become part of an integrated mobility system. They need to operate efficiently, be used collectively and become part of public transport solutions. If autonomous shared services were available to complement the public transport system now, we would need only seven percent of the vehicles that are currently on the roads. This was the conclusion of a simulation study carried out by PTV for the city of Oslo.

The key to creating the right conditions for autonomous vehicles is to already gain an understanding of the impact they are likely to have. This will be the only way of ensuring that we can exploit their full potential for sustainable mobility in the future. 